\title{
Cyclic bisbibenzyls induce growth arrest and apoptosis of human prostate cancer PC3 cells
}

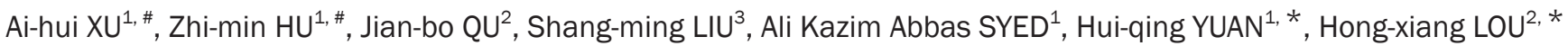 \\ ${ }^{1}$ Department of Biochemistry and Molecular Biology, School of Medicine, Shandong University, Ji-nan 250012, China; ${ }^{2}$ Department of \\ Natural Product Chemistry, School of Pharmaceutical Sciences, Shandong University, Ji-nan 250012, China; ${ }^{3}$ Department of Histology \\ and Embryology, School of Medicine, Shandong University, Ji-nan 250012, China
}

\begin{abstract}
Aim:To investigate the cytotoxic effects of four cyclic bisbibenzyls, Riccardin C (Ric), Pakyonol (Pak), Marchantin M (Mar), and Plagiochin E (Pla) against chemoresistant prostate cancer PC3 cells.

Methods: Cell growth was assayed by MTT method, and apoptotic related protein Bcl-2 and Bax, poly(ADP-ribose) polymerase (PARP) were examined by Western blotting. Cell cycle and apoptosis of PC3 cells were evaluated with flow cytometry and morphologic examinations.

Results: The four compounds inhibited proliferation and elicited cell death in a dose- and time-dependent manner with $\mathrm{IC}_{50}$ values of $3.22 \mu \mathrm{mol} / \mathrm{L}$ for Ric, $7.98 \mu \mathrm{mol} / \mathrm{L}$ for Pak, $5.45 \mu \mathrm{mol} / \mathrm{L}$ for Mar, and $5.99 \mu \mathrm{mol} / \mathrm{L}$ for Pla, respectively. Furthermore, exposed to these chemicals caused a decrease in the antiapoptotic protein $\mathrm{Bcl}-2$ and an increase in proapoptotic Bax expression. PARP cleavage and caspase-3 activity were also observed.

Conclusion: The results suggest that cyclic bisbibenzyls could be used for the development of novel therapeutic chemicals against prostate cancer.
\end{abstract}

Keywords: cyclic bisbibenzyls; liverworts; apoptosis; prostate carcinoma; PC3 cells; Bcl-2; poly(ADP-ribose) polymerase

Acta Pharmacologica Sinica (2010) 31: 609-615; doi: 10.1038/aps.2010.37; published online 26 April 2010

\section{Introduction}

The development of androgen-independent prostate cancer is mainly believed to be apoptosis resistant, and is accompanied by increased proliferation and survival of cells within primary or metastatic tumors ${ }^{[1]}$. There is no effective therapy available at present for curing or controlling the androgen-independent stage of prostate cancer due to the high recurrence of apoptosis resistance. Cytotoxic chemotherapies or radiotherapy has shown to provide improvement of the patient's condition. However, significant side effects are observed from these treatments. The clinical impact of advanced prostate cancers has led to the exploration of novel treatment modalities as well as new anticancer agents.

Naturally occurring products are gaining more attention

\footnotetext{
\# These authors contributed equally to this work.

* To whom correspondence should be addressed.

E-mail louhongxiang@sdu.edu.cn (Hong-xiang LOU) lyuanhq@sdu.edu.cn (Hui-qing YUAN)

Received 2009-12-02 Accepted 2010-03-08
}

in chemotherapy because they are a rich source of antitumor compounds. A variety of secondary plant metabolites have been developed as antitumor drugs, including taxol and camptothecin and their analogues ${ }^{[2-5]}$. Liverworts has been shown to produce various types of natural products including terpenoids, phenolic compounds, and cyclic bisbibenzyls $^{[6-12]}$. The cyclic bisbibenzyls are particularly interesting because they constitute a class of natural products with unique structural frameworks, and exhibit a diversity of biological activities as indicated by their antimicrobial and antifungal activities $^{[8,9,13,14]}$, cytotoxicity ${ }^{[6,7,15,16]}$, induction of apoptosis ${ }^{[17]}$, reversal of multidrug resistance ${ }^{[18]}$, and inhibitory effects on 5-lipoxygenase, cyclooxygenase and DNA polymerase ${ }^{[8,19]}$. Whether the bisbibenzyls exhibit inhibitory effects on prostate cancer cells remained to be investigated. In collaborative work aimed at developing safe and effective natural products for prostate cancer chemotherapy, we investigated whether bisbibenzyls could induce apoptosis of chemo-resistant PC3 cells. In our preliminary study, we found that the bisbibenzyls inhibited cell growth and promoted apoptosis of androgenindependent prostate cancer cells. 


\section{Materials and methods Chemicals}

The cyclic bisbibenzyls riccardin C (Ric $)^{[10]}$, pakyonol (Pak $)^{[12]}$, marchantin M (Mar) ${ }^{[9]}$, and plagiochin E (Pla) ${ }^{[11]}$ (Figure 1) were isolated from Ric Asterella angusta, Plagiochasm intermedium L, Asterella angusta, and Marchantia polymorpha L, respectively. Purification and structure determination were described previously ${ }^{[9-12]}$. These compounds were prepared in dimethyl sulfoxide (DMSO) and stored as small aliquots at $-20^{\circ} \mathrm{C}$

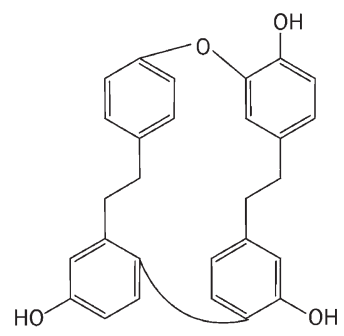

Riccardin C (Ric)

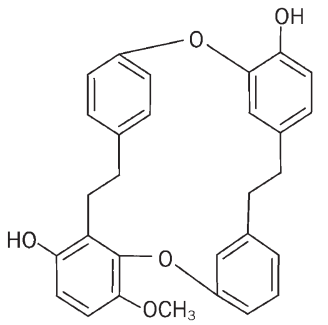

Marchantin M (Mar)

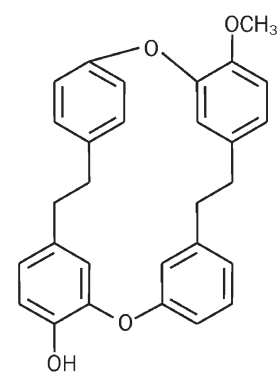

Pakyonol (Pak)

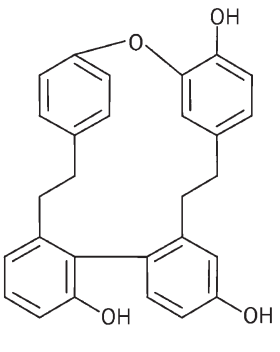

Plagiochin E (Pla)
Figure 1. Structures of bisbibenzyls.

\section{Cell culture and treatments}

Human prostate cancer PC3 cells (The Cell Bank of Chinese Academy of Sciences, Shanghai) were cultured in RPMI-1640 medium (HyClone) supplemented with $10 \%$ fetal bovine serum (HyClone). Human hTERT-RPE1 cells (a gift from Dr Chang-jun ZHU, School of Medicine, Shandong University) were cultured in DMEM-F12 medium (HyClone) containing $10 \%$ fetal bovine serum (HyClone). The cells were maintained in $5 \% \mathrm{CO}_{2}$ at $37{ }^{\circ} \mathrm{C}$ until reaching approximately $50 \%-70 \%$ confluence, and then treated with different amounts of chemicals as indicated. DMSO alone was used as the control vehicle.

\section{MTT assay}

Cells $\left(1 \times 10^{4}\right.$ per well) were seeded in 96 -well culture plates, and challenged with the indicated concentrations of Ric, Pak, Mar or Pla. After 24, 48 and $72 \mathrm{~h}$ of treatment, cells were incubated with $10 \mu \mathrm{L}$ MTT ( $5 \mathrm{mg} / \mathrm{mL}$, 3-[4,5-dimethylthiazol-2-yl]2,5-diphenyltetrazolium bromide; Sigma, St Louis, MO, USA) for $4 \mathrm{~h}$ and the cell growth response to the chemicals was detected by measuring the absorbance at $570 \mathrm{~nm}$ on a plate reader (Bio-Rad, USA). Three replicates were used for each treatment.

\section{Detection of apoptosis}

Apoptotic cells were detected by morphologic observation and flow cytometry analysis. For morphologic evaluation, the PC3 cells were cultured in 24-well plates and incubated under the conditions described above. After treatment with the indicated chemicals for $24 \mathrm{~h}$, cells were stained with $10 \%$ Giemsa or with propidium iodide (PI, $100 \mu \mathrm{g} / \mathrm{mL}$ ) and Hochest33342 $(20 \mu \mathrm{g} / \mathrm{mL})$ followed by washing with phosphate buffered solution (PBS). The results were analyzed using light microscopy (NIKON). In addition, quantification of apoptotic cells was determined by flow cytometry. Briefly, following incubation with the indicated concentrations of Ric, Pak, Mar or Pla, cells were harvested, washed with PBS, and fixed with cold ethanol $(70 \%, v / v)$. Cells were resuspended in PBS, $0.1 \%$ saponin and $1 \mu \mathrm{g} / \mathrm{mL}$ RNase A (Sigma), incubated for $20 \mathrm{~min}$ at $37^{\circ} \mathrm{C}$, and stained with $25 \mu \mathrm{g} / \mathrm{mL}$ PI (Sigma). Cell cycle distribution and the sub-G1 population were then evaluated.

\section{Western blot assay}

After treatment with Ric, Pak, Mar or Pla, cells were washed twice with ice-cold PBS and a cell lysate was prepared as previously described ${ }^{[20]}$. Protein concentrations were determined using the Bradford method. Cell extracts containing $50 \mu \mathrm{g}$ of proteins were resolved by SDS-polyacrylamide gel and electro-transferred onto a nitrocellulose membrane (PALL). The membranes were blocked with $5 \%$ non-fat milk in TBST buffer (20 mmol/L Tris- $\mathrm{HCl}, 137 \mathrm{mmol} / \mathrm{L} \mathrm{NaCl}$, and $0.1 \%$ Tween 20, $\mathrm{pH}$ 8.0) for $1 \mathrm{~h}$ at room temperature prior to incubation with specific antibodies to Bcl-2, Bax, caspase-3, PARP, or $\beta$-tubulin (all antibodies from Santa Cruz Biotechnology) overnight at $4{ }^{\circ} \mathrm{C}$. After washing and reaction with horseradish peroxidase conjugated anti-mouse IgG (Zhongshan, Beijing, China), or anti-rabbit IgG (ZB-2301, Zhongshan, Beijing, China) secondary antibodies for $1 \mathrm{~h}$, the membranes were washed with TBST buffer three times and the proteins on the membrane were detected using an enhanced chemiluminescence substrate (ECL, Millipore).

\section{Caspase- 3 activity analysis}

Caspase-3 activity was analyzed using a caspase- 3 activity assay kit (Beyotime, China). Cells were treated with the indicated chemicals for $24 \mathrm{~h}$ and cell lysates were prepared according to the manufacturer's instructions. The activity of caspase- 3 was detected at a wavelength of $405 \mathrm{~nm}$ following addition of the substrate to the lysate. A standard curve was formed by detecting the absorbance of standard samples at different concentrations.

\section{Statistical analysis}

The data are presented as the mean \pm SD of at least three independent experiments: MTT assay, caspase-3 activity analysis, flow cytometry. The statistical significance of difference between the control and treated groups was determined by a paired $t$-test. $P<0.05$ was considered statistically significant. 


\section{Results}

Bisbibenzyls are cytotoxic to PC3 cells

We first investigated the effects of bisbibenzyls on androgeninsensitive PC 3 cell proliferation. The kinetic changes of cell growth, as measured by MTT assays, were monitored in cells exposed to bisbibenzyls at 5, 10, 20, and $50 \mu \mathrm{mol} / \mathrm{L}$ for 24,48 , and $72 \mathrm{~h}$. Viability increased in control cells with increasing exposure time, while pretreatment of the cells with bisbibenzyls resulted in a significant reduction in cell proliferation. In response to treatment with each compound, the concentration response analysis revealed that cell viabilities were decreased at $5,10,20$, and $50 \mu \mathrm{mol} / \mathrm{L}$ by $0.1 \%, 3.4 \%, 84.1 \%, 88.3 \%$ repres- sion for Ric; $9.8 \%, 23.2 \%, 42.1 \%$, 56.8\% for Pak; $23.2 \%, 29.1 \%$, $39.5 \%, 76.8 \%$ for Mar; and 19.1\%, 24.2\%, 31.2\%, 89.1\% for Pla, respectively, after $24 \mathrm{~h}$ of treatment (Figure 2A-2D). This suggests that bisbibenzyls' inhibition on cell growth became detectable at $24 \mathrm{~h}$. The apparent inhibitory activities were observed in cells treated with bisbibenzyls up to $48 \mathrm{~h}$ and $72 \mathrm{~h}$ of exposure in a dose-dependent manner (over 50\% repression at $5 \mu \mathrm{mol} / \mathrm{L}$ for each compound). All bisbibenzyls exerted dramatic cytotoxic effects on PC3 cells at the lower concentration and showed almost complete inhibition at concentrations up to $50 \mu \mathrm{mol} / \mathrm{L}$. The concentration response data demonstrated $50 \%$ growth inhibitions $\left(\mathrm{IC}_{50}\right)$ at $3.22 \mu \mathrm{mol} / \mathrm{L}$ for Ric,
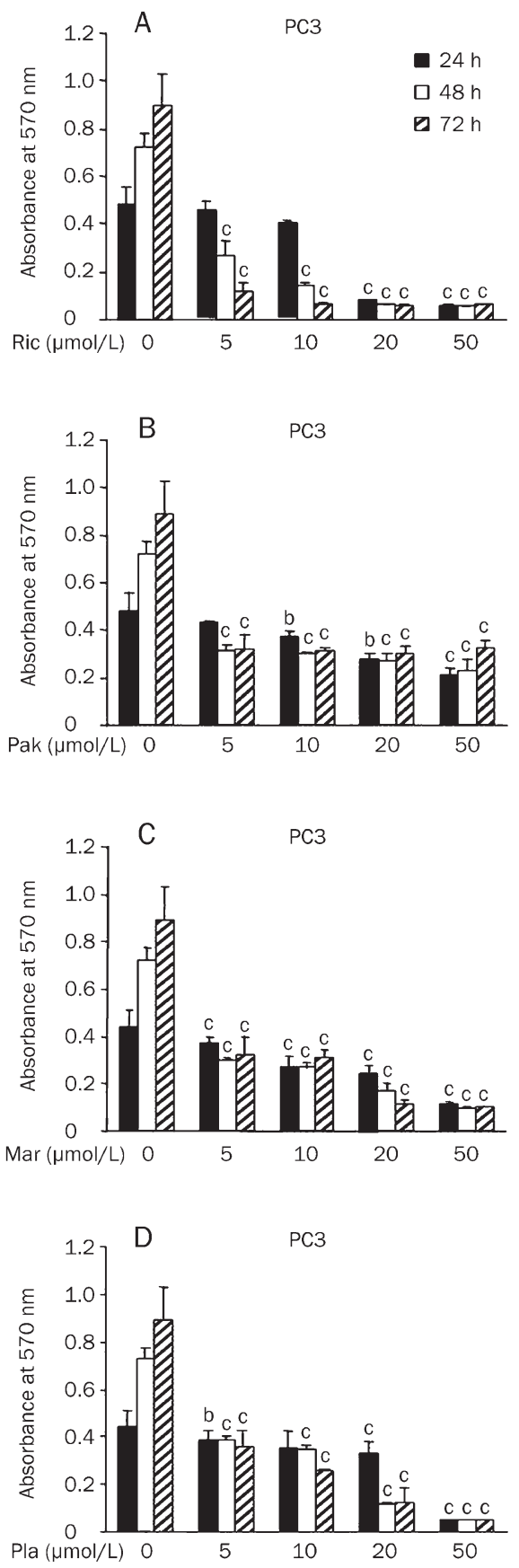
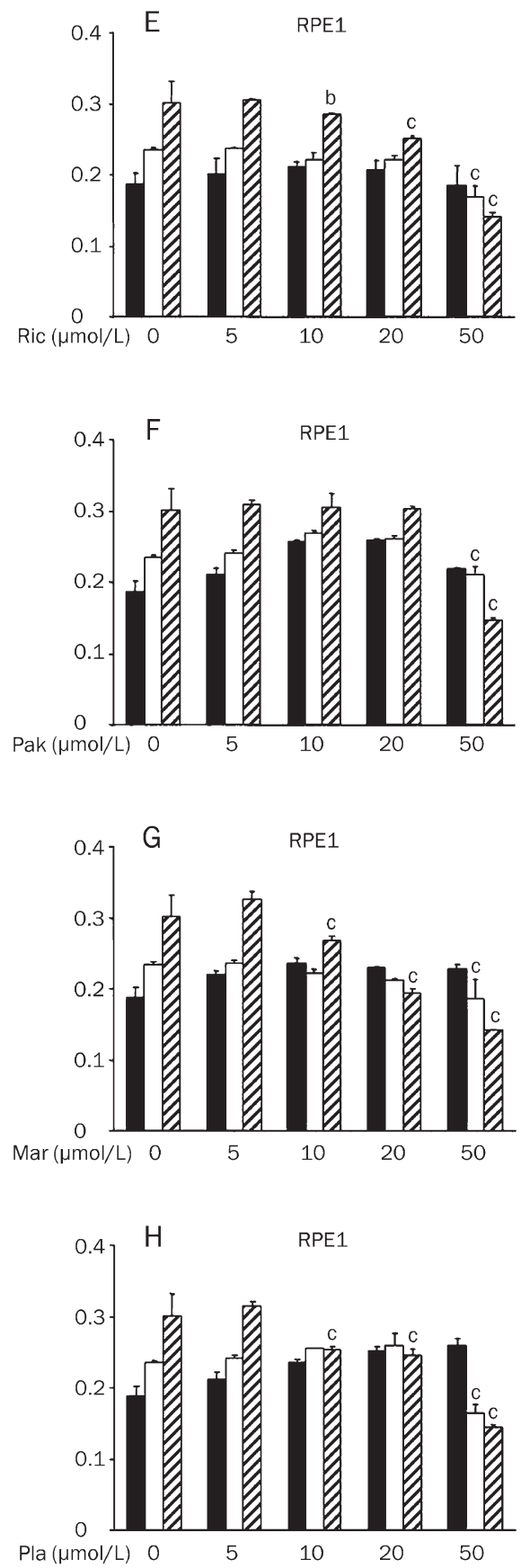

Figure 2. Effect of bisbibenzyls on cell proliferation. Changes in PC3 cell viability after treatment with increasing concentrations of Ric (A), Pak (B), Mar (C), and Pla (D) for 24, 48, 72 h. Changes in RPE1 cell viability after treatment with Ric (E), Pak (F), Mar (G), and Pla (H). Cell viability was assayed by MTT. Control cells were treated with equal volumes of DMSO. Results are expressed as mean \pm standard error (SEM), and are representative of at least three separated experiments. $\left({ }^{b} P<0.05,{ }^{c} P<0.01\right.$ vs 0 $\mu \mathrm{mol} / \mathrm{L}$ group). 
$7.98 \mu \mathrm{mol} / \mathrm{L}$ for Pak, $5.45 \mu \mathrm{mol} / \mathrm{L}$ for Mar, and $5.99 \mu \mathrm{mol} / \mathrm{L}$ for Pla, respectively, after $48 \mathrm{~h}$ of treatment. All four compounds were cytotoxic against PC 3 cells, with $\mathrm{IC}_{50}$ in the range of 3-8 $\mathrm{mol} / \mathrm{L}$. In contrast, the sensitivity of normal human retina pigment epithelial cells (RPE1) to bisbibenzyls was much lower under the present experimental conditions, with the four bisbibenzyls only having cytotoxic effects on RPE1 cells at the higher dose $(50 \mu \mathrm{mol} / \mathrm{L})$ following $72 \mathrm{~h}$ of treatment as shown in Figure 2E-2H. Bisbibenzyls may have weak cytotoxicity to normal cells, but facilitate the death response in cancer cells. These results (Figure 2) indicated that bisbibenzyls proportionally inhibited PC3 cell proliferation in a doseand time-dependent manner.

Additionally, we examined cytotoxic effects of these chemicals on cells by propidium iodide (PI) and Hochest33342 staining under a fluorescence microscope. Figure 3 showed the dose-response results. Cells were treated with four bisbibenzyls as indicated concentrations for $24 \mathrm{~h}$, cell growth response was found to be decreased in cells treated with chemicals in a dose-dependent manner. Moreover, depending on the intensity of the challenge, late apoptosis and dead cells were noticeable after bisbibenzyls treatments at cytotoxic doses used as indicated in Figure 3. All together, these data indicated that bisbibenzyls-induced cell death may be due to the induction of apoptosis in PC3 cells.

\section{Bisbibenzyls induce apoptotic response}

The importance of apoptosis resistance in the proliferation and survival of PC3 cells has been emphasized by many independent studies. We next defined whether the bisbibenzylsrelated cell death was a result of the induction of apoptosis by examining morphological changes and employing a flow cytometry assay. When PC3 cells were stained with Giemsa and examined under a microscope, the morphology of the control cells remained unchanged. However, significant morphological changes such as shrunken cell bodies, condensed DNA and apoptotic bodies were observed in cells treated with four bisbibenzyl compounds after $24 \mathrm{~h}$ in a dose-dependent manner as shown in Figure 4A. Apoptotic cells increased in bisbibenzyl-treated cells at all concentrations tested. Induction of apoptosis by four bisbibenzyls was consistent with their growth inhibitory effects.

Furthermore, the extent of apoptotic cells was also quantified using a flow cytometry assay. Consistent with their effect on cell growth inhibition, bisbibenzyl treatment for $48 \mathrm{~h}$ resulted in significant increases in the sub- $\mathrm{G}_{1}$ cell population in PC3 cells (Figure 4B), and the observed increase was accompanied by dose-dependent induction of apoptosis. The percent of apoptotic cells in the vehicle control was low $(0.05 \% \pm$ $0.01 \%)$, while the number of apoptotic cells was increased in cells exposed to the four bisbibenzyls. The total percent of apoptotic cells resulting from bisbibenzyls treatment was $6.21 \%$ for Ric $(10 \mu \mathrm{mol} / \mathrm{L}), 9.40 \%$ for Pak $(10 \mu \mathrm{mol} / \mathrm{L}), 51.62 \%$ for Mar $(10 \mu \mathrm{mol} / \mathrm{L})$, and $14.46 \%$ for Pla $(10 \mu \mathrm{mol} / \mathrm{L})$. At cytotoxic doses, these inhibitors induced considerable cell apopto-
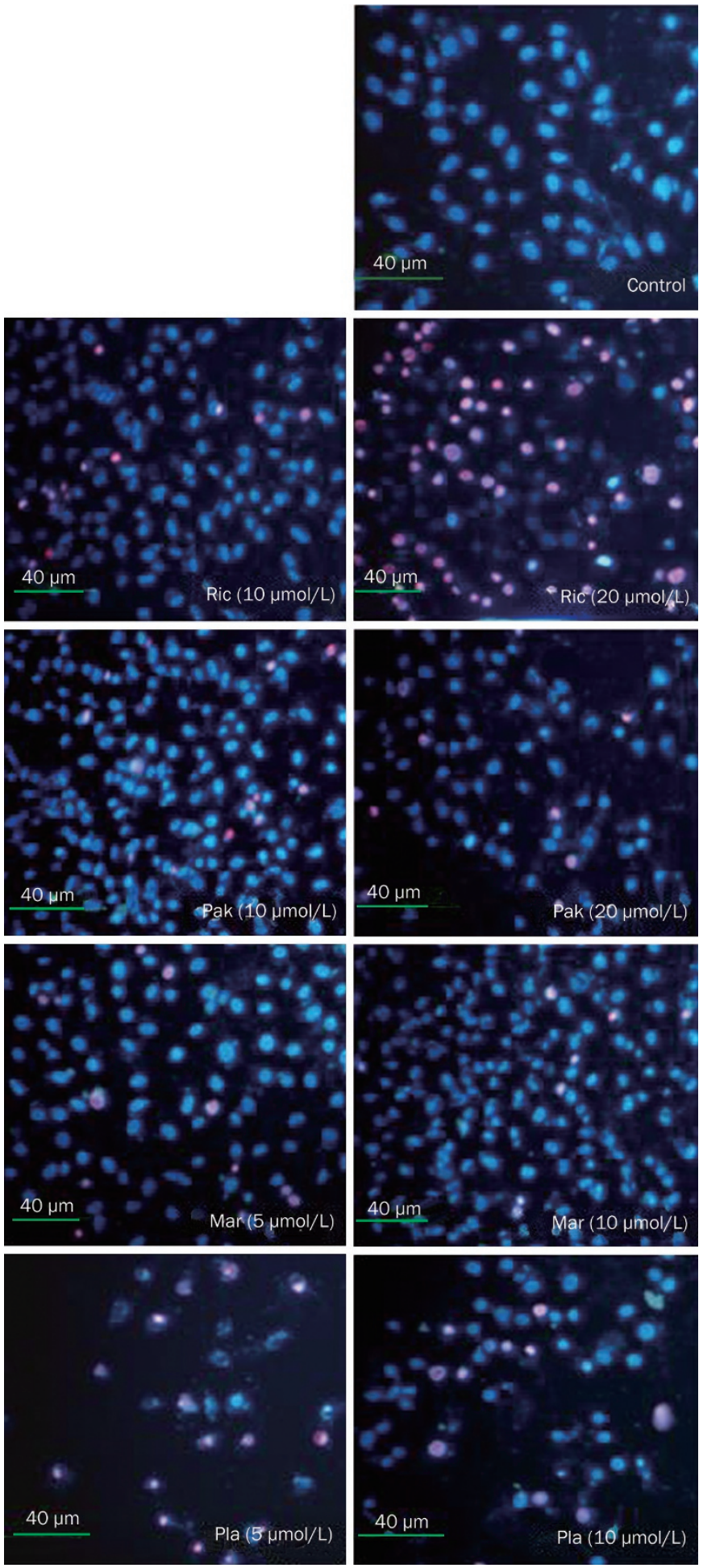

Figure 3. Cytotoxic effect of bisbibenzyls on PC3 cells. Cells were treated with vehicle alone or specified concentrations of Ric, Pak, Mar, and Pla for $24 \mathrm{~h}$. Morphological changes were examined by staining with Hoechest 33342 and propidium iodide, and the images were observed and photographed under a fluorescence microscopy.

sis and it seemed that PC3 cells were more sensitive to the Mar treatment. 

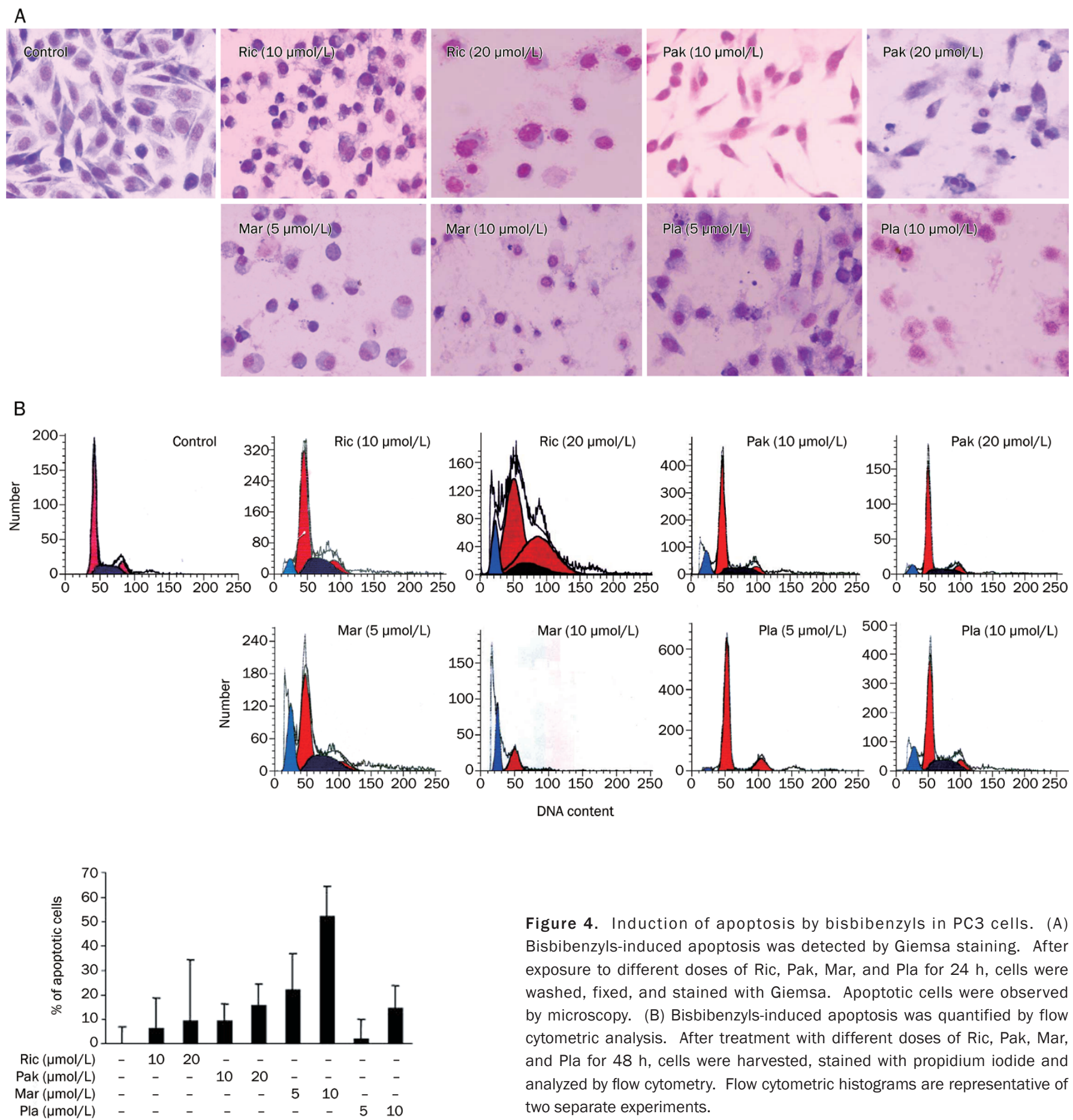

Figure 4. Induction of apoptosis by bisbibenzyls in PC3 cells. (A) Bisbibenzyls-induced apoptosis was detected by Giemsa staining. After exposure to different doses of Ric, Pak, Mar, and Pla for $24 \mathrm{~h}$, cells were washed, fixed, and stained with Giemsa. Apoptotic cells were observed by microscopy. (B) Bisbibenzyls-induced apoptosis was quantified by flow cytometric analysis. After treatment with different doses of Ric, Pak, Mar, and Pla for $48 \mathrm{~h}$, cells were harvested, stained with propidium iodide and analyzed by flow cytometry. Flow cytometric histograms are representative of two separate experiments.

Bisbibenzyls modulate expression levels of apoptosis-related proteins in PC3 cells

Apoptosis is characterized by changes in expression and activity of several apoptotic markers. The first indication came from the expression changes of the Bax and Bcl-2 protein levels using Western blot analysis. The proapoptotic marker Bax markedly increased with exposure to the four bisbibenzyls, whereas the expression level of Bcl-2 in the treated cells was significantly decreased (Figure 5A). Caspases are responsible for cell apoptosis and activation of the caspase cascade leading to poly (ADP-ribose) polymerase (PARP) cleavage is regarded as a major pathway in apoptosis induction ${ }^{[21]}$. Therefore, we determined the effect of bisbibenzyls on the activation of caspase- 3 and PARP by Western blot analysis. The results indicated that no detectable changes in the level of procaspase 3 were observed in bisbibenzyl-treated cells at lower doses, while the decreased expression of procaspase 3 was noticeable in Ric-, Mar-, and Pla-treated cells at $20 \mu \mathrm{mol} / \mathrm{L}, 10 \mu \mathrm{mol} / \mathrm{L}$, 
A

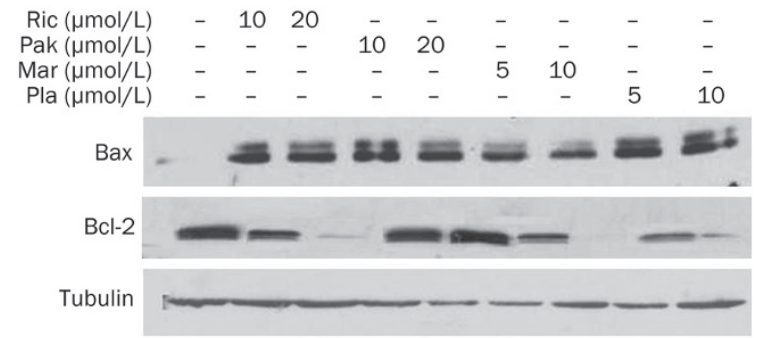

B

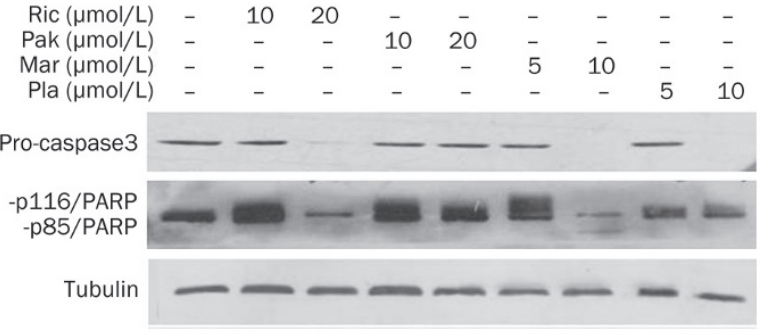

C

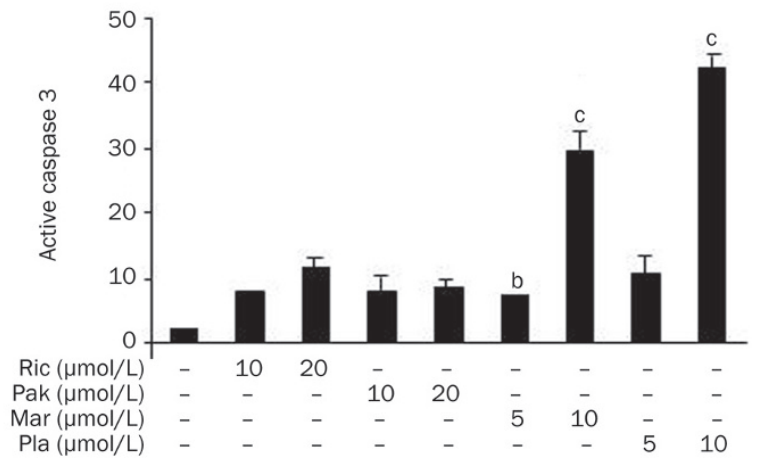

Figure 5. Effects of bisbibenzyls on the expressions of apoptosis-related genes in PC3 cells. (A) Western blot analysis of Bax and $\mathrm{Bcl}-2$ protein levels in whole cell lysates from PC3 cells treated with vehicle, or indicated chemicals. (B) Whole cell lysates from cells treated with control vehicles, or each bisbibenzyl were prepared for Western blot analysis of caspase 3 and PARP cleavage. $\beta$-tubulin served as an internal control to monitor protein loading and transferring efficiency. (C) Cell lysates from vehicle- or bisbibenzyls-treated cells were collected for analysis of caspase 3 activity. $\left({ }^{\mathrm{b}} P<0.05,{ }^{\mathrm{c}} P<0.01\right)$.

and $10 \mu \mathrm{mol} / \mathrm{L}$, respectively (Figure 5B). Consistent with caspase- 3 activity, the native $116 \mathrm{kDa}$ PARP protein was found to be cleaved into its characteristic $85 \mathrm{kDa}$ fragment after bisbibenzyl treatment (Figure 5B). A significant decrease in the native form of PARP or PARP cleavage was obvious and corresponded to $20 \mu \mathrm{mol} / \mathrm{L}, 10 \mu \mathrm{mol} / \mathrm{L}$, and $10 \mu \mathrm{mol} / \mathrm{L}$ exposure to Ric, Mar and Pla treatment, respectively. To confirm that the apoptotic effect of bisbibenzyls was associated with caspase- 3 activation, we analyzed caspase- 3 activity based on enzymatic assay. The level of caspase-3 activation was substantially higher in cells exposed to the chemicals for $24 \mathrm{~h}$ than the cells exposed to the vehicle control (Figure 5C). These results indicated that bisbibenzyl-related cell death was associated with changes in apoptosis-related gene expressions and activities.

\section{Discussion}

A variety of bisbibenzyls that are characteristic constituents of liverworts have been demonstrated to have antimicrobial and antifungal activities ${ }^{[22,23]}$. Recently, bisbibenzyls are gaining much more attention for their cytotoxic effects and anti-tumor activities $^{[7,15-18]}$. The current work represents the beginning of collaborative efforts to identify novel natural products that target androgen-independent prostate cancer. Our data showed that four compounds, Ric, Pak, Mar, and Pla, belonging to the bisbibenzyl family were able to cause a concentration- and time-dependent inhibition of PC3 cell growth. Treatment with these compounds resulted in accumulation of apoptotic cells in a dose-dependent manner, which suggested that inhibition of cell growth by bisbibenzyls was associated with the induction of cell apoptosis. Based on the percentage of apoptotic cells induced by the bisbibenzyls, we found that PC3 cells were more sensitive to Ric, Mar, and Pla than to Pak at the concentrations tested. This was supported by the types of cellular morphological changes observed including shrunken cell bodies, condensed DNA, and apoptotic bodies. Induction of apoptosis by bisbibenzyls was further investigated by monitoring changes in the typical apoptotic markers Bcl-2, the survival molecule, and Bax, caspase-3, and PARP, the apoptotic molecules, in a dose-dependent manner. Not surprisingly, each bisbibenzyl induced the strong expression of Bax protein, and the level of expression of Bcl-2 decreased significantly. Furthermore, bisbibenzyl-related apoptosis was accompanied by activation of caspase- 3 and cleavage of PARP. This data unequivocally supported bisbibenzyls as novel and potent apoptosis inducers for androgen-independent prostate cancer cells.

However, the ability of each bisbibenzyl to induce apoptosis and up-regulate apoptotic markers was not identical. It seems that the effect of Pak (as one hydroxyl group in its structure is methylated) was weaker than the others tested in the study. For example, treatment with Ric, Mar, and Pla induced massive cell death and significant morphological changes at 10 $\mu \mathrm{mol} / \mathrm{L}$ (Figure $4 \mathrm{~A})$, whereas a high dose of Pak $(20 \mu \mathrm{mol} / \mathrm{L})$ was necessary for an obvious inhibition in cell growth. Moreover, Ric, Mar, and Pla were more potent than the same concentration of Pak for activation of caspase-3 and induction of cleavage of PARP (Figure 5). Whether the $\mathrm{OH}$ group and the adjacent oxygen bridge are essential features for the inhibitory effects of bisbibenzyls remains to be clarified. Efforts are being made to identify the structural determinants of the initiation of apoptosis by bisbibenzyls. In addition, the results in Figure 5 showed that PARP cleavage, as an indicator of caspase activation and apoptosis, was not very remarkable since there was a significant increase in PI-positive cells after treatment with bisbibenzyls (Figure 2, 4). Using z-VAD-fmk, a general caspase inhibitor, to reduce caspase activity might provide evidence of the importance of caspase signal pathway in bisbibenzyls-mediated effects on PC3 cells. Interestingly, bisbibenzyls-induced apoptosis was still significant in the presence of caspase inhibitor (data not show). This result suggests that other pathways may be involved in bisbibenzyls- 
induced apoptosis in addition to caspase signal pathway and should be investigated in the future.

With the progression of prostate cancer, hormone refractory tumors have become resistant to conventional approaches including radiotherapy and chemotherapy ${ }^{[24]}$. The identification of bisbibenzyl compounds as novel apoptosis inducers at low concentration levels $(<10 \mu \mathrm{mol} / \mathrm{L})$ provides evidence for their potential use in the treatment of androgen-independent prostate cancer. In addition, treatment with bisbibenzyl compounds has been shown to dramatically suppress androgen and androgen receptor (AR) signaling in LNCaP cells, suggesting that bisbibenzyls could also be useful for androgendependent prostate cancer therapy (data not show). Many lines of evidence have shown that even in hormone-refractory prostate cancer, AR signaling is important for the growth and survival of cells ${ }^{[25,26]}$. Therefore, the ability of bisbibenzyls to inhibit cell growth and induce apoptosis could also be important for hormone-refractory prostate cancer therapy.

\section{Acknowledgements}

This work was supported by the National Natural Science Foundation of China (30772594), Shandong Provincial Key Research Foundation (2006GG1102023) and Shandong Scientific Technology Program (2008GG10002042).

\section{Author contribution}

Hui-qing YUAN and Ai-hui XU designed research; Ai-hui XU and Zhi-min HU performed research; Jian-bo QU and Hong-xiang LOU contributed new reagents and analytic tools; Shang-ming LIU, Hui-qing YUAN, and Ai-hui XU analyzed data; Ai-hui XU, Ali Kazim Abbas SYED, and Hui-qing YUAN wrote the paper.

\section{Abbreviations}

PI, propidium iodide; PARP, poly(ADP-ribose) polymerase; Ric, Riccardin C; Pak, Pakyonol; Mar, Marchantin M; Pla, Plagiochin $\mathrm{E} ; \mathrm{AR}$, androgen receptor

\section{References}

1 Gurumurthy S, Vasudevan KM, Rangnekar VM. Regulation of apoptosis in prostate cancer. Cancer Metastasis Rev 2001; 20: $225-$ 43.

2 Lee $\mathrm{KH}$. Anticancer drug design based on plant-derived natural products. J Biomed Sci 1999; 6: 236-50.

3 Kucuk 0. Chemoprevention of prostate cancer. Cancer Metastasis Rev 2002; 21: 111-24.

4 Barnes S. Role of phytochemicals in prevention and treatment of prostate cancer. Epidemiol Rev 2001; 23: 102-5.

5 Singh RP, Dhanalakshmi S, Agarwal R. Phytochemicals as cell cycle modulators: a less toxic approach in halting human cancers. Cell Cycle 2002; 1: 156-61.

6 Asakawa Y, Toyota M, Taira Z, Takemoto T, Kido M. Riccardin A and Riccardin B, two novel cyclic bibenzyls possessing cytotoxicity from the liverwort Riccardia multifida (L.) S. Gray. J Org Chem 1983; 48: 2164-7.

7 Scher JM, Burgess EJ, Lorimer SD, Perry NB. A cytotoxic sesquiterpene and unprecedented sesquiterpenebisbibenzyl compounds from the liverwort Schistochila glaucescens. Tetrahedron 2002; 58: 7875-82.

8 Nagashima F, Momosaki S, Watanabe Y, Toyota M, Huneck S, Asakawa Y. Terpenoids and aromatic compounds from six liverworts. Phytochemistry 1996; 41: 207-11.

9 Qu JB, Xie CF, Guo HF, Yu WT, Lou HX. Antifungal dibenzofuran bis(bibenzyl)s from the liverwort Asterella angusta. Phytochemistry 2007; 68: 1767-74.

10 Xie CF, Qu JB, Wu XZ, Liu N, Ji M, Lou HX. Antifungal macrocyclic bis(bibenzyls) from the Chinese liverwort Ptagiochasm intermedlum $\mathrm{L}$. Nat Prod Res 2010; Feb 23: 1-6. [Epub ahead of print].

11 Niu C, Qu JB, Lou HX. Antifungal bis[bibenzyls] from the Chinese liverwort Marchantia polymorpha L. Chem Biodivers 2006; 3: 34-40.

12 Wang FQ, Lou HX. Chemical studies on the constituents of Plagiochasm intermedium L. Acta Pharmacol Sin 2000; 35: 587-91.

13 Baek SH, Phipps RK, Perry NB. Antimicrobial chlorinated bibenzyls from the liverwort Riccardia marginata. J Nat Prod 2004; 67: 71820.

14 Scher JM, Speakman JB, Zapp J, Becker H. Bioactivity guided isolation of antifungal compounds from the liverwort Bazzania trilobata (L.) S.F. Gray. Phytochemistry 2004; 65: 2583-8.

15 Kodama M, Shiobara Y, Sumitomo H, Matsumura K, Tsukamoto M, Harada C. Total syntheses of marchantin A and riccardin B, cytotoxic bis(bibenzyls) from liverworts. J Org Chem 1988; 53: 72-7.

16 Shi YQ, Zhu CJ, Yuan HQ, Li BQ, Gao J, Qu XJ, et al. Marchantin C, a novel microtubule inhibitor from liverwort with anti-tumor activity both in vivo and in vitro. Cancer Lett 2008; 276: 160-1

17 Shi YQ, Liao YX, Qu XJ, Yuan HQ, Li S, Qu JB, et al. Marchantin C, a macrocyclic bisbibenzyl, induces apoptosis of human glioma A172 cells. Cancer Lett 2008; 262: 173-82.

18 Shi YQ, Qu XJ, Liao YX, Xie CF, Cheng YN, Li S, et al. Reversal effect of a macrocyclic bisbibenzyl plagiochin $E$ on multidrug resistance in adriamycin-resistant K562/A02 cells. Eur J Pharmacol 2008; 584: 66-71.

19 Asakawa Y, Bors W, Franck U, Michel C, Muller-Jakic B, Nenninger A, et al. Effect of marchantins and related compounds on 5-lipoxygenase and cyclooxygenase and their antioxidant properties: a structureactivity relationship study. Phytomedicine 1995; 2: 113-7.

20 Yuan HQ, Kong F, Wang XL, Young C, Hu XY, Lou HX. Inhibitory effect of acetyl-11-keto- $\beta$-boswellic acid on androgen receptor by interference of $\mathrm{Sp} 1$ binding activity in prostate cancer cells. Biochem Pharmacol 2008; 75: 2112-21.

21 Nicholson DW, Ali A, Thornberry NA, Vaillancourt JP, Ding CK, Gallant $\mathrm{M}$, et al. Identification and inhibition of the ICE/CED-3 protease necessary for mammalian apoptosis. Nature 2002; 376: 37-43.

22 Asakawa Y. Biologically active substances found in Hepaticase. Stud Nat Prod Chem 1988; 2: 277-92.

23 Asakawa Y. Recent advances in phytochemistry of bryophytesacetogenins, terpenoids and bis(bibenzyl)s from selected Japanese, Taiwanese, New Zealand, Argentinean and European liverworts. Phytochemistry 2001; 56: 297-312.

24 Feldman BJ, Feldman D. The development of androgen-independent prostate cancer. Nat Rev Cancer 2001; 1: 34-45.

25 Zegarra-Moro OL, Schmidt LJ, Huang HJ, Tindall DJ. Disruption of androgen receptor function inhibits proliferation of androgenrefractory prostate cancer cells. Cancer Res 2002; 62: 1008-13.

26 Liao XB, Tang SQ, Thrasher JB, Griebling TL, Li B. Small-interfering RNA-induced androgen receptor silencing leads to apoptotic cell death in prostate cancer. Mol Cancer Ther 2005; 4: 505-15. 